\title{
Urgences
}

\section{" Au fond, je sais, il n'y a que la poésie " \\ Gaston Miron, À bout portant. Correspondance de Gaston \\ Miron à Claude Haeffely 1954-1965, Montréal, Leméac, 1989, $174 \mathrm{p}$.}

\section{André Gervais}

Numéro 25, octobre 1989

Multiples de Hamlet

URI : https://id.erudit.org/iderudit/025547ar

DOI : https://doi.org/10.7202/025547ar

Aller au sommaire du numéro

Éditeur(s)

Urgences

ISSN

0226-9554 (imprimé)

1927-3924 (numérique)

Découvrir la revue

Citer ce compte rendu

Gervais, A. (1989). Compte rendu de [" Au fond, je sais, il n'y a que la poésie " /

Gaston Miron, À bout portant. Correspondance de Gaston Miron à Claude

Haeffely 1954-1965 , Montréal, Leméac, 1989, 174 p.] Urgences, (25), 83-88.

https://doi.org/10.7202/025547ar d'utilisation que vous pouvez consulter en ligne.

https://apropos.erudit.org/fr/usagers/politique-dutilisation/ 


\section{deliveline}

"Au fond, je sais, il n'y a que la poésie"

À bout portant. Correspondance de Gaston Miron à Claude Haeffely 1954-1965, Montréal, Leméac, 1989, $174 \mathrm{p}$.

Gaston Miron est - et, poétiquement, comme Mallarmé, ne sera peut-être que - l'homme d'un seul livre 1: L'homme rapaillé (première édition: Montréal, PUM, 1970). Oui, bien sûr, mais que faites-vous de Deux sangs (1953) en collaboration avec Olivier Marchand - et de Courtepointes (1975)? La réponse est dans l'édition revue et augmentée de L'homme rapaillé (Paris, Maspéro, 1981), ou le recueil de 1975 trouve sa place dans la réorganisation et l'amplification des cycles ( 4 La vie agonique », "La batèche», etc.) et de ce qui vient avant ou après, comme le premier - recueil de «débutants» et plaquette inaugurale d'une nouvelle maison d'édition de la poesie: l'Hexagone avait déjà trouvé la sienne ("Au pays du son bleu ») dans la première édition.

Et les autres poèmes - recyclage du vieux stock ou écriture nouvelle - ainsi que les autres textes en prose viendront naturellement y trouver leur place. Car il y a chez Miron, dans l'un ou l'autre cas, un travail - public et privé, parlé et écrit - de (re)formulation, de densification, d'énonciation et d'écologie textuelles liées. Déjà, dans la lettre du 5 août
1958 (p. 102): «il est entendu que la poésie fut pour moi une aventure où j'ai laissé ma peau. Et que je n'ai rien produit depuis mes 27 ans. Ceci étant posé, je t'envoie deux poèmes que j'ai déterrés de mes papiers du temps de la dérision. Ma dérision. Et LA dérision. C'est une contribution dont tu peux être étonné, soit! $\gg$ Depuis mes 27 ans: depuis 1955 , cela faisant donc trois ans. Ou encore (1 er novembre 1958 , p. 110): «Tu comprends, c'est malcommode de toujours vivre sur du vieux, sur des stocks de quatre ou cinq ans, lesquels s'épuisent. Que veux-tu, j'ai fini ma carrière littéraire à 27 ans. Dans la pagaille, la confusion, le laisser-aller. »

Un travail où la variante, voire la variation, devient une donnée essentielle. Déja, dans la lettre du 24 mars 1958 (p. 79): «En ce qui concerne "Des pays et des vents", il y a peut-être cent versions; je ne sais laquelle est authentique et laquelle ne l'est pas. Ce n'est plus un poème. "Cette dernière phrase, qui n'est apparemment qu'elliptique (ce n'est plus un poème inédit, ayant déjà été publié en 1955), va, en fait, beaucoup plus loin. Miron est probablement le poète d'ici qui a le plus exhibé, même par defaut, son travail d'écriture, en publiant dans des revues et des journaux, dans des anthologies et dans les recueils satellites, plusieurs états, souvent incomplets, d'un texte qui ne trouvera telle place précise dans tel cycle, par exemple, qu'en bout de ligne, dans les deux éditions du livre (L'homme rapaillé), elles-mêmes nullement definitives, sinon, comme le Grand Verre de Marcel Duchamp, définitivement 
inachevées ${ }^{2}$. Les poèmes, ainsi rétrogradés à l'état de "lambeaux », de «guenilles», de "débris» (21 février 1956 , p. 48 et 49 ), de "petits chancres personnels (26 novembre 1956, p. 55) - chancre: dérision du chant, lésion de la "voie* —, de "quelques exercices dits poétiques " (7 avril 1958, p. 82) ou de «quelques griffonnages $\gg$ (17 avril 1958, p. 89), pauvres entre les « pauvres » (p. 55) et en danger comme «la vie tout court en danger * (15 décembre 1954, p. 32), dévalués avant d'être lus vraiment, ne peuvent être qu'en position précaire - entre le brouillon perpétuel et le recyclage radical, entre la proferation et l'ecriture - face à "CECI, le nonpoème $s$.

Or, les 52 lettres qui viennent tout juste d'être publiées, on le constate dejà par les quelques passages déjà cités, éclairent plutôt spectaculairement cette situation.

On sait toute limportance des lettres - et du journal - de Kafka dans l'économie de son œuvre presque entièrement posthume. Ici, on saura l'importance, publiée du vivant de l'auteur et avec son assentiment, selon une "édition brute * (dit Pierre Filion, éditeur, si je comprends bien, dans les deux sens du mot) et dans une présentation précieusement sobre, de cette correspondance à sens unique les lettres d'Haeffely n'ayant pas encore été retrouvées - étalée sur une douzaine d'années. Autant on peut et on a pu avoir tendance à penser que Miron a, comme on dit, tout donné en 1970 , s'épuisant depuis à parler, voire à déparler selon sa légende - c'est le "cabotin», le «pitre» (Mallarmé encore) dans le personnage - autant on ne pourra qu'être surpris de constater que les trajectoires de l'ecriture privée et de l'écriture publiée se confondent souvent, et qu'il y a nécessaire enchevêtrement, ici et là, de la problématique, voire de la dialectique du " poème * et du * nonpoème ». Et il faudra aussi rappeler qu'il n'a jamais cessé d'écrire, tout comme Duchamp, aura dit une légende qui aujourd'hui ne tient plus, n'a pas mis de côté l'art (en 1923 , lui qui vivra jusqu'en... 1968) pour devenir un joueur d'échecs 4 .

En effet, le premier paragraphe de la lettre du 5 septembre 1958 (p. 104-105) est tout a fait explicite sur la question de l'engagement:

"Dès qu'un poète veut faire de la politique, il doit s'affilier à un parti, et alors, en tant que poete, il est perdu. " Voilà ce que Gœthe écrivait, et qui est toujours vrai. Voila pourquoi je suis toujours, plus que jamais, sans espoir de retour, à cent lieues de la poésie. Celle-ci est affaire de sensibilité avant tout. La politique est d'abord une idée révolutionnaire, et partant, une idee efficace. André Breton a établi, encore hier dans un interview qu'il accordait aux Nouvelles litteraires, une distinction fort à propos et fort habile au sujet de l'engagement du poète. Sa poésie ne doit être assujettie qu'aux imperatifs propres à la poésie, mais par contre le poète se discrédite si, comme homme, il ne s'engage pas. Mais cette situation du poète et de sa poesie conduit à des voies de cache-cache pour lesquelles je n'al aucune sympathie. Mieux vaut sacrifier un tableau au profit de l'autre. Comme ça, tout est clair.

D'un côté, donc, l'action, de l'autre, la création. D'un côté, la lutte, de l'autre, la littérature. 
D'un côté, « je réussis quand même à vivre grâce à une ligne d'action à laquelle je m'accroche désespérément. Je deviens activités, gestes, êtres et objets. » (p. 32); de l'autre, «L'amour fut, dans la projection de ma vie, la pierre d'angle, la raison de vivre unique. Et cet amour n'est jamais apparu a la ligne d'horizon.» (20 juillet 1958 , p. 100). Ou encore, comme le lie - et le lit cette longue lettre du 19 janvier 1960 (p. 130):

C'est en 1955 que j'ai pris
conscience du CCF, du
Parti Socialiste et progres-
siste, je trouvais donc une
structure à tout mon back-
ground, à mes inquiétudes
profondes. Si, par la suite,
j'ai relié mon échec amou-
reux à l'écriture, c'était in-
consciemment une ma-
nière de liquider l'écriture,
les autres m'emprisonnant
dans une attente littéraire,
alors que je me foutais
bien de la littérature, de
Breton, Michaux, etc. et
pas du tout de Lénine,
Marx, Chardin. II n'en
demeure pas moins que:
Poésie égale Femme
egale Amour, justement
parce que la Poésie a
coïncidé, de façon acci-
dentelle chez moi, avec
mes amours. 5

D'un côté, donc, "mes vraies obsessions qui étaient plutôt d'ordre idéologique et d'action * (19 janvier 1960 , p.129); de l'autre, «Je dois m'avouer, en toute sincérité et objectivité, que l'écriture est bien stérilisée ${ }^{6}$ chez moi, que la poésie est bien morte, bel et bien, il n'y a plus rien à chercher de ce côté-là. En dépit de mes dénégations des dernières années, j'espérais secrètement qu'il n'en était rien » (15 novembre 1959, p. 123). Autant Miron, durant toutes ces années, à Montréal ou à Paris, ne cessera en quelque sorte d'aspirer au - et d'être aspiré par le - «non-poème*, ne cessera de quitter - et d'être quitté par le «poème», autant il aura posé, dès le début, que «Au fond, je sais, il n'y a que la poésie. * (16 novembre 1954 , p. 27) - «t pourtant, le langage est peut-être le seul et dernier honneur. * (p. 55) - , autant il n'aura de cesse d'y revenir, d'y avoir, de fait, toujours eté: «je ne suis plus revenu pour revenir/je suis arrivé à ce qui commence * dit clairement "L'homme rapaillé", poème liminaire du livre eponyme. Et « ce qui commence", c'est bien autant un certain homme québécois ("me voici en moi comme un homme dans une maison/qui s'est faite en son absence"), un certain projet québécois ( je n'ai jamais voyagé/vers autre pays que toi mon pays » et «un homme reviendra/d'en dehors du monde * est-il écrit dans «Pour mon rapatriement»), que, naturellement, un livre - ce livre: *Du train où vont les choses, c'est comme si je voyais des pierres se détacher d'un édifice avant même que celui-ci soit édifié. (12 décembre 1965, p. 168). «Pour mon rapatriement " et *L'homme rapaille»: de 1956 à 1970, la trajectoire est, littéralement, happee, entre action et poésie, entre angoisse et poésie (cf. p. 22), entre poésie et maladie * accès poétique [...] assimilé à maladie» (p. 89) -, entre poésie et amour: l'aliénation, de tous les côtés - comme dans « rapatriement » ou dans "rapaille», justement -, guette (p. 85, 131, 155). Il est certain, désormais, que l'« anthropoete $*$, nom qui apparaît, sauf erreur, dans le titre d'une interview de $1972^{7}$ et qui est repris dans le prière d'insérer de la quatrième de couverture d'À bout portant, s'il unit par le *-po-»- ou par la peau : «il est entendu que la poésie fut pour moi une aventure 
ou j'ai laissé ma peau » - l'homme et le poète, n'a été, côté action, par un renversement maintenant inevitable, qu'un poete en trop. Le terme - et l'homme - de référence est bien, malgré les dénégations répétées, malgré le «malgré moi ${ }^{8}$, (le) poète, et non (l')homme d'action.

De la même façon, Duchamp, proposant en 1963 dans une interview une definition du ready-made ("a work of art without an artist to make it ), le ready-made qui avait pourtant été défini dans une note, en 1913 - avant même d'être nommé "ready-made» -, comme étant une cuvre qui ne soit pas d'art ( Peut-on faire des œuvres qui ne soient pas “d'art”? - ), garde et le terme art et le terme artiste comme points de référence, appuyant sur la disparition tantôt de l'un, tantôt de l'autre.

On voit comment, de « Lhomme agonique», cycle, à *I a pauvreté anthropos», poème (le premier vers est " Ma pauvre poésie en images de pauvres »), par exemple, un titre en lit un autre. Comment un titre, décalqué sur un autre "La povreté Rutebeuf", poème du XIII' ${ }^{\mathrm{P}}$ siècle -, peut aller chercher un nom: "Amnésique Miron (cf. p. 63 et 113), «Archaïque Miron* (cf. p. 119), ou encore celui d'«Amérique * (p. 63, 66, 72-73) ${ }^{9}$. Comment le nom d'une maison d'édition («Hexagone») peut, par sa première lettre et par une syllabe qu'on ajoute à sa dernière, être a la fois le noud et l'ici d'une problématique.

Dans la signature ("miron") de l'autoportrait fait en 1954 et reproduit sur la première de couverture, le "o* est lié au * $n *$, et ces deux lettres sont séparées des trois premieres, liees elles aussi. Cet « 0 » est l'icône de l'ovale du visage, comme le lien entre le wo» et le «n» est l'icône du «6 " qui revient cinq fois dans la constitution des traits du visage: les yeux (et des lunettes), les ailes du nez, et, juste en surplomb de la signature, le dernier $* 6 * q u i$, on le voit bien maintenant, est en fait l'icône d'un "G*, initiale du prénom. Prénom (six lettres) et nom (cinq lettres) qui se terminent tous deux par le pronom justement dit indéfini, en ce qu'il désigne étymologiquement l'homme (*on * vient du latin "homo ", homme), objet du " poème", autant qu'il participe phoniquement et graphiquement du * nonpoème».

Comment, donc, un recueil de lettres, même partiel, est utile, repositionnant et relançant le questionnement critique.

\section{André Gervais}

L'homme d'un seul livre: comme Montaigne, aussi (et pas vraiment comme Corbiere ou Émile Nelligan, disparus trop vite). Le rapport l'ecriture, au livre, a linstitution, au passage à vide (oisiveté, etc.) ou au passage a l'action (politique, etc.) est ici tout a fait different que, par exemple, chez Hugo ou Francois Charron, hommes des livres nombreux. Montaigne, Mallarme, Miron auront $e u$, auront pris le(ur) temps.

2 Ce n'est pas la premiere fois que j'insiste sur cet aspect du travail mironien. Voir déja le * Post-scriptum vite " des deux numéros que j'aj préparés pour La nouvelle barre du jour (Montréal, $n^{\circ} 182$, octobre 1986 , et $n^{\circ}$ 191 , février 1987) sur la question de l'uavant-texte *. (Pour une définition de ce terme, voir Urgences, $\mathrm{n}^{\circ} \mathbf{1 9}$, janvier 1988, p. 18-19.) Quelques écrivains québecois devaient *établir * eux-mêmes l'avant-texte d'un de leurs textes, texte bref choisi par eux, et proposer quelque réflexion sur son/ leur écriture. J'avais, bien sar, demandé une collaboration a Miron, mais cela, finalement, ne se fit pas. J'ai tenu quand meme, dans ce post-scriptum et pour les raisons que je viens de dire, lui rendre hommage en quelque sorte. Il y a la, évidemment, toute une recherche a mener, qui commence a 
peine. Faut-il ajouter qu'à la même époque, Anne Hébert aura été la première à discuter, vers a vers et presque mot a mot, un de ses plus importants poemes (" Le tombeau des rois *, publié en revue en 1951 avant d'etre repris, en 1953, dans le livre éponyme) et $\mathrm{ga}$ traduction par Frank Scott. Dialogue Hebert-Scott publie, comme l'on sait, en 1960 dans la revue Écrits du Canada français, puis republié en livre, dans une version augmentee essentiellement d'une nouvelle traduction ( $\alpha$ Une traduction ne peut jamais être considérée comme tout à fait terminée, mesme aux yeux du traducteur. écrit ce dernier), chez HMH, en 1970. Sur ces questions, Miron et Hébert peuvent servir de phares, ici, à un travail aujourdhui dit textuel.

Lhomme rapaillé, Montréal, coll. du Prix de la revue Études françaises, PUM, 1970, p. 127. C'est en 1965, année où il écrit la derniere lettre ici publiée a Haeffely, que Miron publie dans la revue Parti pris deux textes fondamentaux: *Un long chemin * (janvier) et * Notes sur le non-poeme et le poème (juin-juillet).

L'homme rapaillé (édition de 1981) contenant des textes ecrits jusqu'en 1975 , voir, entre autres, les poemes publiés dans Liberté, Montréal, n* 120 , novembre-décembre 1978 , no 150 , décembre $1983, n^{\circ} 166$, août 1986 , et $\mathrm{n}^{\circ}$ « Special $101 \& *$, fevrier 1987; dans Estuaire, Montréal, no 30, hiver 1984, et now 32-33, été-automne 1984; dans Poésie 84, Paris, Seghers; dans Maison de la poésie, Saint-Martin-d'Hères (France), no 2-3, janvier 1988. colncidences. Aimé Césaire, par exemple, a rendu bon à rien tout ce que je puis écrire. Aussi, je me ferme la bofte. Je suis un ramassis des échos de ce siecle * (25 février 1958, p. 77 voir, deja, 21 février 1956 , p. 48). Amour/Aimé, ou quand l'intertextua. lité et l'hypertextualité sont aussi vécues comme un échec. Pour une définition de ces termes, voir Ungences, $n^{\circ} 19$, p. 48-49 et 40-42. Il faudra attendre 1970 et le dernier poeme de Lhomme rapaillé (magnifiquement intitule, en regard du afil conducteur de l'hommem, « En une seule phrase nombreuse *) pour en lire une autre version: «Je demande pardon aux poetes que j'ai pilles / - poetes de tous pays, de toutes époques $-/$ je n'avais pas d'autres mots, d'autres écritures / que les vôtres, mais d'une façon, frères / c'est un bjen grand hommage à vous .. La violence du pillage fait entendre, dans l'aveu sil

en est, quelque grand dommage, et le "mais d'une facon $*$, qui désigne ici la torsion de l'ecriture mironienne, n'est plus, comme en 1958 , rabattu sur le scripteur qui ne peut qu'en être réduit au silence, *ramassis * d'alterités: il désigne plutôt l'autonomie et l'autonomination, il g'autodesigne souverainement. " Je demande pardon * et "mais d'une façon * signant alors, a la rime, Gaston Miron.

"Mon élément, c'est ce qui importe, c'est l'action, non la création. [...] JE NE SUIS PAS POËTE.[...] Ma création poetique, c'est d'agir. (16 avril 1958, p. 84). "A cette époque, je me suis preté au jeu, consciemment ou non, parce que la cause de la poésie l'exigeait: la poésie, en 1953, en etait a un point mort. Et de nature, j'avais de la poigne sur les gens, je pouvais pétarader en pleine rue, gueuler devant une assemblee, en somme faire avancer la cause de la poésie. Mais toutes ces qualités ressortis. saient beaucoup plus a l'homme d'action qu'au créateur. * (17 avril 1958 , p. 89). «Quand je fais le compte, je crois que l'Hexagone m'interesse en autant que c'est une action, et comme action. (p. 129). On ne peut âtre plus clair, et courageux, dans la stérilisation des " petits chancres personnels *, dans le chatiment du poete qui fut. Pourtant, ce ne sont pas les invitations a publier qui manquent, des cette epoque: aux Ed. Erta de Roland Giguère en 1956 (cf. p. 49), à l'Hexagone en 1958 (p. 108), au Périscope, petite revue d'Haeffely, justement, «premiere revue francophone internationale (comme l'écrit Miron en 1978 dans le catalogue établi pour les 25 ans de l'Hexagone), encore en 1958 (p. 110), ou tout un numéro - choix de lettres et de poèmes - lui serajt consacré. D'un côté, donc, faire avancer la cause de la poésie; de l'autre, «'avance en poésie.... section de L'homme rapaille ainsi normmée d'après un hémistiche d'un vers d'un poeme publie en 1963.

7 Jacques Picotte: * Rencontre avec Gaston Miron, Anthro-Podte *, Nouveau monde, Montreal, vol. $\mathrm{V}, \mathrm{n}^{\circ} 10,15$ juillet-15 aoat 1972 .

8 Téléphonant a Miron le 15 octobre 1989, deux jours après avoir écrit ce compte rendu, pour des précisions bibliographiques (voir note 3 ), je l'entends me dire a peu près ce qui suit: «j'aime souvent dire que j'ecris malgré moi, que j'ecris hors de moi * et (parlant des annees 1950 et 1960) * cette alienation, c'est quasiment ma langue maternelle.. La bataille contre 
l'aliénation (côté unon-poème *) est aussi, est déjà une bataille dans la langue (côte * poème *): c'est bien «malgré moi », contre ma volonté et "pour ne pas périr * (27 octobre 1954, p. 25) -, que, griffonnant «mes papiers du temps de la dérision •, j'ecris. On aura remarqué l'anagrammatique sas qui va de "papiers * a "pas périr > et inversement.

9 «Amnésique Miron *: dans * Notes sur le non-poème et le poème* (1965), Lhomme rapaille, p. 126. "Archaique Miron *: dans «Epitaphe*, poeme publie dans L'arbre d parales, Flemalle (Belgique), n55, septembre 1985.

\section{Casgrain : 2, Crémazie: 1}

\section{Réflexions autour de l'histoire littéraire et de ses aléas contemporains au Québec}

À lire ce qui s'écrit au Québec sur la littérature québécoise, j’ai de plus en plus l'impression que l'histoire littéraire d'ici n'appartient pas vraiment à la littérature ou, pour le dire autrement, que la littérature québécoise, dans l'esprit tout au moins d'un bon nombre de ceux qui se sont donné pour tâche de retracer les lignes de son parcours, ne trouve pas vraiment - pas encore? - sa place dans la vaste histoire des littératures de ce monde. Quelque chose achoppe. Je sens de plus en plus nettement le malaise et les effets pervers d'un non-dit, d'une sorte d'inavouable douloureux qui mine la substance même du discours.

Ce que j'avance ne vise ni les théories ni les méthodes. De ce côté, les choses bougent. Pour s'en convaincre, il n'y a qu'a lire les actes du colloque sur l'histoire litteraire, tenu à l'Université Laval, en octobre 1986 , et qui viennent dêtre publiés aux Presses de l'Université Laval ${ }^{1}$.
Mes propos sont d'un autre ordre. Ils appartiennent à ce que l'on pourrait appeler l'observable des pratiques, tant celles de l'écriture que celles de la lecture. Pratiques que révèlent les rares publications destinées à un public débordant la communauté des chercheurs et des spécialistes de l'histoire litteraire.

Plus concrètement, et il n'y a qu'à regarder autour de soi, les travaux relevant de l'histoire littéraire (études, biographies ${ }^{2}$, etc.) n'intéressent grosso modo, que ceux qui s'y emploient. Ils n'intéressent guère les lecteurs (du moins, c'est ce que semblent penser les auteurs savants et les éditeurs de littérature générale). Ils n'intéressent pas plus - le fait mérite qu'on s'y arrête - les écrivains québécois ${ }^{3}$. Pourtant, ailleurs, on reaffirme limportance des jeux complexes et vitaux, pour la littérature, de la filiation. A une question portant sur ses "maîtres" en littérature, l'écrivain espagnol Camilo José Cela, prix Nobel 1989, répondait récemment: «Tous ceux qui ont ecrit en espagnol avant moi, absolument tous, sans aucune exception. . Et il ajoutait ceci en quoi je crois aussi très fortement: «La littérature est une culture, une course à relais, il n'y a pas chez elle de generration spontanée ou de science infuse. 4 Autrement dit, comme l'écrivait Mallarmé ou Valéry (j'oublie): La littérature naît de la littérature. Bref, qui s'intéresse à la littérature ne peut espérer avancer sans prendre en considération les acquis de ses prédécesseurs.

On parle peu, ici, d'histoire littéraire ou, si l'on en parle, c'est d'une manière gênée, réactivant à même les idées et hypothèses, les regrets d'un lointain mais toujours tenace échec. On laisse entendre (ces choses-là ne se disent jamais à voix haute) qu'il est dommage que 